\title{
Inverse Percolation to Quantify Robustness in Multiplex Networks
}

\author{
Edwin Montes-Orozco $\mathbb{D},{ }^{1}$ Roman-Anselmo Mora-Gutiérrez $\mathbb{D}{ }^{2}$ \\ Bibiana Obregón-Quintana $\mathbb{D}^{\mathbb{D}}{ }^{3}$ Sergio-G. de-los-Cobos-Silva $\mathbb{D}^{\mathbb{D}},{ }^{4}$ Eric A. Rincón-García $\mathbb{D}^{4}{ }^{4}$ \\ Pedro Lara-Velázquez $\mathbb{D}^{4},{ }^{4}$ and Miguel A. Gutiérrez-Andrade $\mathbb{D}^{4}{ }^{4}$ \\ ${ }^{1}$ Posgrado en Ciencias y Tecnologías de la Información, Universidad Autónoma Metropolitana Iztapalapa, Mexico City, Mexico \\ ${ }^{2}$ Departamento de Sistemas, Universidad Autónoma Metropolitana Azcapotzalco, Mexico City, Mexico \\ ${ }^{3}$ Facultad de Ciencias, Universidad Nacional Autónoma de México, Mexico City, Mexico \\ ${ }^{4}$ Departamento de Ingeniería Eléctrica, Universidad Autónoma Metropolitana Iztapalapa, Mexico City, Mexico
}

Correspondence should be addressed to Edwin Montes-Orozco; emontes@xanum.uam.mx

Received 26 March 2020; Revised 20 August 2020; Accepted 1 October 2020; Published 31 October 2020

Academic Editor: Vincent Labatut

Copyright (c) 2020 Edwin Montes-Orozco et al. This is an open access article distributed under the Creative Commons Attribution License, which permits unrestricted use, distribution, and reproduction in any medium, provided the original work is properly cited.

\begin{abstract}
Inverse percolation is known as the problem of finding the minimum set of nodes whose elimination of their links causes the rupture of the network. Inverse percolation has been widely used in various studies of single-layer networks. However, the use and generalization of multiplex networks have been little considered. In this work, we propose a methodology based on inverse percolation to quantify the robustness of multiplex networks. Specifically, we present a modified version of the mathematical model for the multiplex-vertex separator problem (m-VSP). By solving the m-VSP, we can find nodes that cause the rupture of the mutually connected giant component (MCGC) and the large viable cluster (LVC) when their links are removed from the network. The methodology presented in this work was tested in a set of benchmark networks, and as case study, we present an analysis using a set of multiplex social networks modeled with information about the main characteristics of the best universities in the world and the universities in Mexico. The results show that the methodology presented in this work can work in different models and types of 2- and 3-layer multiplex networks without dividing the entire multiplex network into single-layer as some techniques described in the specific literature. Furthermore, thanks to the fact that the technique does not require the calculation of some structural measure or centrality metric, and it is easy to scale for networks of different sizes.
\end{abstract}

\section{Introduction}

1.1. Complex Networks and Robustness. A complex network is a network with nontrivial topological characteristics, which do not occur in simple networks, such as degree distributions, high local cohesiveness (measured through the clustering coefficients, etc.), community structures, and hierarchical structures [1].

In complex networks, robustness is a measure of the capacity of complex systems versus specific external or internal events to preserve a relatively normal behavior. The robustness of the network is understood as its ability to keep most nodes together after a disturbance [1].

Robustness in complex networks can be considered as an inverse percolation process [2] because the percolation theory models the process of placing nodes in a random $n$ dimensional network with some probability $p$ and thus predict the sudden formation of a giant component (GC). Therefore, the inverse percolation process can be considered as the time when the rupture (separation) of the GC occurs.

1.2. Multiplex Networks. In recent years, the study of multilayer networks has been emphasized, thanks to the fact that most real systems have structures with multiple types of links or interactions between nodes [3], for example, multimodal transport systems, biological systems, social networks, and numerous modes of communication [4].

On the other hand, multiplex networks are a particular class of multilayer networks, which were introduced to 
better model complex real-world systems [5-7]. The main characteristic of multiplex networks is that all the nodes in each layer are replicated in other layers, and there is a direct link between each replica node to denote the relationship.

Formally, let GP $=\left(\mathscr{G}_{\alpha}, \mathscr{C}\right) \forall \alpha \in\{1, \ldots, M\}$ be a multiplex network where:

(i) $\mathscr{G}_{\alpha}=\left(X_{\alpha}, E_{\alpha}\right)$ is a monoplex network called layer $\alpha$, where $X_{\alpha}$ and $E_{\alpha}$ are the set of nodes (it is important to mention that, in multiplex networks, each node belongs to all the $M$ layers.) and the links in layer $\alpha$, respectively.

(ii) $\mathscr{G}=\left\{E_{\alpha \beta} \subseteq X_{\alpha} \times X_{\beta} ; \alpha, \beta \in\{1, \ldots, M\}, \alpha \neq \beta\right\}$ is the set of interconnections between nodes in different layers. The elements of $\breve{G}$ are called cross layers, and the elements of each $E_{\alpha}$ are called intralayer connections of GP.

For these types of networks, a question that has been the subject of considerable recent research is as follows: if we work with a multilayer or multiplex network, how robust is the communication with the failure or elimination of certain nodes? In response to this question, it is known that the elimination of certain nodes in a single-layer can cause cascading failures.

Furthermore, as in the case of single-layer networks, if the nodes are removed randomly, the effect on the transmission is very slight, but if the nodes to be removed are chosen carefully, they can cause the entire system to collapse.

1.3. Approach. In this work, we present a methodology to quantify robustness in multiplex networks based on the inverse percolation process applied to the mutually connected giant component (MCGC) and the large viable cluster (LVC). We analyze this methodology through a set of instance networks, and as a case study, we present a set of networks modeled with information about the main characteristics of 377 universities that belong to the 500 best in the world (QSRanking networks) and 60 universities that belong to the Mexican Higher Education System (MHES networks).

It is worth mentioning that the QSRanking networks were modeled using the information available in the QS Intelligence Unit [8], where the main characteristics studied are academic reputation, employer reputation, college students, college appointments, international teachers, and international students.

On the other hand, the MHES networks were modeled using information available in the EXECUM [9] repository of the National Autonomous University of Mexico (UNAM), where some of the main characteristics are number of professors with recognition from the National System of Researchers (SNI) of the Mexican National Council of Science and Technology (CONACYT), professors with Masters or Ph.D., registered students, accredited academic programs, publications in SCOPUS, publications in indexed journals, postgraduate programs incorporated into CONACYT's National Registry of Quality Graduate Programs (PNPC), among others [10].
The idea of modeling 2- and 3-layer multiplex networks is based on the advantage of analyzing the most important nodes based on the information of 2 or 3 years. Thus, it obtains an identification based on a multicriteria analysis that reduces the disadvantages of using only one layer.

\section{Related Work}

Currently, there is no consensus about the definition of robustness in complex networks; however, there are some works that analyze these concepts [11-14].

In some works, such as [15-17], the authors define several measures to quantify robustness in single-layer networks, which are based on the identification of the most vulnerable nodes in the network, and estimate the importance of a disturbance or failure.

For example, in the work of Ren et al. [18], the authors describe that node removal algorithms are inefficient in certain conditions and propose a simple and efficient link removal strategy called Hierarchical Power Iterative Normalized Cut (HPI-Ncut). On the other hand, Jianhua et al. [19], show the design of two optimal mixed-integer nonlinear programming schemes to investigate the optimal robustness of free-scale single-layer networks.

On the other hand, Morone and Makse [20] present a methodology based on the mapping of the optimal percolation problem in random networks, intending to identify the minimum set of influential nodes (spreaders).

In the work of Montes-Orozco et al. [21], the authors present a methodology based on inverse percolation that finds the set of nodes that disconnect the network in order to quantify the robustness in single-layer networks.

For multilayer networks, Shekhtman et al. [22] present a survey about the vulnerability and resilience of systems with two or more interdependent networks; specifically, they show the consequences of a disturbance or attack on a particular node of a specific network and describe that this is the case when some nodes have an essential role in at least two networks (layers).

Furthermore, there are some works about cascade failures in multilayer networks $[16,23,24]$; however, they only focus on targeted attacks and not in system failures. For example, Kleineberg et al. [24] describe how an attack can bring cascading failures inside multilayer networks, and in the study developed by Schneider et al. [16], the authors describe how the attacks to certain node into a specific layer affect the nodes connected to it in other layers.

In the work of Baxter et al. [25], the authors develop a theory of sparse multiplex networks, which makes it possible to find the mutually connected giant component (MCGC) in a 2-layer multiplex network with arbitrary correlations between connections of different types. Here, the authors found that the correlations between the overlapping and nonoverlapping bonds markedly change the phase diagram of the system, leading to multiple hybrid phase transitions.

Bianconi [26] posed the following question: are there metrics that can be used to design robust multilayer networks? Some authors have tried to find a measure to answer this problem [27]; however, the question remains open 
$[4,28]$. On the other hand, in the work of Vaknin et al. [28], the authors present a study based on the propagation of attacks located in multiplex networks, where some experiments are executed using different values for the assortativity index and the percolation threshold.

In the work of Cozzo et al. [4], the authors analyze the triadic relationships for the generalization of transitivity between layers and clustering coefficient in multiplex networks. Also, the authors show how the structure of the multiplex networks adds a new fundamental intransitivity parameter.

Stella [29] presents a study about the strength of the lexicon in the face of the progressive failure of words with multiplex network attacks. Here, the author indicates that the MCGC is robust to many targeted attacks that, instead, dramatically disrupted the large viable cluster (LVC).

Osat et al. [30] present an analysis based on optimal percolation, where the authors describe that approximating the optimal percolation solution in a multiplex network with valid solutions for monolayer networks extracted from the multiplex can have serious consequences in the characterization of the true robustness of the system.

Finally, Montes-Orozco et al. [31] present a methodology to identify the spreader nodes of COVID-19 through the inverse percolation approach in 5-layer multiplex networks using the analysis of the relationship between sociocultural and economic characteristics with the number of infections and deaths caused by the COVID-19 virus in different countries.

\section{Materials and Methods}

In this section, we present the main characteristics of benchmark networks, the strategy that we used to model the MHES networks, and the development of the multiplexvertex separator problem (m-VSP).

3.1. Benchmark Networks. It is important to mention that because robustness in multiplex networks is a new topic, there is no reference set to compare the results. Therefore, to verify if $\mathrm{m}$-VSP works correctly for several network models, we use four 3-layer multiplex networks with different characteristics of the reference set given by De Domenico et al. [32].

The networks selected for this work are CKM Physicians Innovation Multiplex Social (CKM) [33], Hepatitis C Virus Multiplex Genetic (Hepatitis C) [34], Krackhardt High Tech Multiplex Social (Krackhardt) [35], and Lazega Law Firm Multiplex Social (Lazega) [36].

Next, we present Table 1, which shows the number of links and nodes for each layer in each benchmark network, where the identifiers are 11 for layer 1,12 for layer 2 , and 13 for layer 3.

At this point, it is important to mention that, although benchmark networks have few links, we use them to verify that our methodology works in different models of multiplex networks. Therefore, we present the numerical results for the main structural metrics calculated in all the benchmark networks.

In order to facilitate the understanding of the results about the structural metrics of networks, we briefly present some fundamental concepts:

(i) Clustering coefficient [37]: it quantifies how much a node is interconnected with its neighbors, where two nodes are neighbors if there is a link that joins them. For nondirected graphs, the clustering coefficient for a node $\left(n_{i}\right)$ is calculated in the following way:

$$
C_{i}=\frac{2\left|E^{\prime}\right|}{k_{i}\left(k_{i}-1\right)}: E^{\prime} \subset E,
$$

where $k_{i}$ is the degree of the node $n_{i}$ and $E^{\prime}$ is the subset of links that connect any pair of nodes in the neighborhood of node $n_{i}$. For this paper, we say that a node $n_{a}$ belongs to the neighborhood of node $n_{i}$ if there is a link between $n_{i}$ and $n_{a}$.

(ii) Average degree [38]: it is a measure that divides the number of links by the number of nodes.

(iii) Average path length [39]: it defines the average number of steps that must be traveled through the shortest path for all possible pairs of nodes.

(iv) Diameter of the network [40]: it is given by the longest route of all the shortest routes between any pair of nodes.

(v) Density [41]: measures the connectivity of the network at the global level, and it is calculated as

$$
\frac{\text { current connections }}{\text { potential connections }}
$$

where "potential connections" (PC) are calculated as

$$
P C=\frac{N(N-1)}{2},
$$

with $N$ as the total number of nodes in the network and "current connections" are the number of links that exists in the network.

In Table 2, we present the numerical values for each characteristic: components, diameter, path length, size of MCGC, and size of LVC. For each characteristic, there are three columns, 11,12 , and 13 that include the information of layers 1,2 , and 3 , respectively.

The information provided in Tables 1 and 2 describes the main characteristics of the selected networks.

For example, according to Table 1, the Hepatitis C network has 106 nodes in each layer and 80, 43, and 2 links in layers 1, 2, and 3, respectively. Besides, according to Table 2, this network has short diameters $(4,4$, and 2$)$ and short average path lengths $(2,2$, and 1.3$)$. 
TABle 1: Main characteristics for benchmark networks.

\begin{tabular}{|c|c|c|c|c|}
\hline \multirow{2}{*}{ Network } & \multicolumn{4}{|c|}{ Nodes } \\
\hline & 11 & 12 & 13 & 11,12 and 13 \\
\hline CKM & 449 & 498 & 423 & 246 \\
\hline Hepatitis C & 80 & 43 & 2 & 106 \\
\hline Krackhardt & 145 & 79 & 20 & 21 \\
\hline Lazega & 717 & 399 & 726 & 71 \\
\hline
\end{tabular}

TABLE 2: Characteristics of benchmark multiplex networks.

\begin{tabular}{lccccccccccc}
\hline \multirow{2}{*}{ Network } & \multicolumn{3}{c}{ Components } & \multicolumn{3}{c}{ Diameter } & \multicolumn{3}{c}{ Path length } & \multicolumn{2}{c}{ Size of MCGC } \\
& 11 & 12 & 13 & 11 & 12 & 13 & 11 & 12 & 13 & 11,12 , and 13 & 11,12, and 13 \\
\hline CKM & 35 & 19 & 22 & 7 & 9 & 14 & 3.1 & 3.4 & 3.9 & 105 & 80 \\
Hepatitis C & 25 & 62 & 103 & 4 & 4 & 2 & 2 & 2 & 1.3 & 804 \\
Krackhardt & 1 & 1 & 1 & 3 & 3 & 4 & 1.3 & 1.6 & 3 & 21 & 71 \\
Lazega & 1 & 3 & 1 & 3 & 5 & 4 & 1.8 & 2.2 & 1.7 & 21 \\
\hline
\end{tabular}

On the other hand, in order to verify that our methodology is easily scalable for large multiplex networks, in the following section, we show the modeling of 172 - and 3-layer multiplex networks, in which each has a greater number of links in every single layer.

3.2. QSRanking and MHES Networks. As mentioned above, we present a case study analysis of the QSRanking and MHES networks. For these networks, each layer represents a different year, where a node represents each university and the links between the nodes are created using the following strategy based on the Mahalanobis distance.

Mahalanobis distance measures a way to determine the similarity between two variables. In contrast with the Euclidean distance, Mahalanobis distance takes into account the correlation between the random variables.

Formally, the Mahalanobis distance between two random variables with the same probability distribution $x$ and $y$ with covariance matrix $\Sigma$ is defined as

$$
d_{m}(x, y)=\sqrt{(x-y)^{T} \Sigma^{-1}(x-y)} .
$$

In our case, the Mahalanobis distance can be considered as follows: based on the different characteristics, we need to measure the similarity between two universities and thus relate those that are similar. For example, assuming that, for each university, only 2 characteristics are considered (number of students and number of professors), and with these data, we can build a vector $x_{i}=\left(x_{1 i}, x_{2 i}\right)^{T}$ for each university $i$.

If we consider that the first characteristic (students) has values between 100 and 200, while the second (teachers) is between 15 and 25, then we use the Euclidean distance:

$$
d_{e}\left(x_{1}, x_{2}\right)=\sqrt{\left(x_{11}-x_{12}\right)^{2}+\left(x_{21}-x_{22}\right)^{2}} .
$$

Since the differences in the first characteristic are greater than in the second, less importance is given to the second characteristic. For this reason, we prefer to add the statistics of the data to the distance measure, weighting according to its variance: the variables with less variance will have more importance than those with greater variance. In this way, we intend to equalize the importance of all the characteristics in the final result.

Therefore, the expression is as follows:

$$
d_{2}\left(x_{1}, x_{2}\right)=\sqrt{\left(\frac{\left(x_{11}-x_{12}\right)}{\sigma_{1}}\right)^{2}+\left(\frac{\left(x_{21}-x_{22}\right)}{\sigma_{2}}\right)^{2}},
$$

where $\sigma_{i}$ is the standard deviation of the component $i i$ of the measurement vectors.

Otherwise, in vectorial notation,

$$
d_{e}\left(x_{1}, x_{2}\right)=\sqrt{\left(x_{1}-x_{2}\right)^{T} S^{-1}\left(x_{1}-x_{2}\right)},
$$

where $S$ is a matrix whose elements on the diagonal are $s_{i i}=\sigma_{i}^{2}$.

However, the expression above has a problem that most of the characteristics are not independent, that is, each one depends on the other since it is more likely that when a university has more students, it will have a greater number of professors. So, to incorporate the dependency between the two variables, we can substitute the diagonal matrix $S$ by the covariance matrix $\Sigma$ :

$$
d_{m}\left(x_{1}, x_{2}\right)=\sqrt{\left(x_{1}-x_{2}\right)^{T} \Sigma^{-1}\left(x_{1}-x_{2}\right)},
$$

which is the Mahalanobis distance.

Then, based on the above, the relationships of the universities are given by the number of characteristics in which the universities are similar, and their quantification is obtained as follows:

(1) The Mahalanobis distance [42] between each pair of universities is calculated.

(2) The median of Mahalanobis distances is calculated.

(3) For each pair of universities with a lower distance than the median, a link is added. 
It is essential to mention that the information provided by ExECUM is divided into two sets: aggregated and disaggregated data. We use both sets of information to analyze the MHES.

In order to better understand the results shown in the following sections, we present the identifiers used for each QSRaking and MHES network:

(1) Each monoplex QSRanking and MHES network represents a different year, where the QSRanking networks have an index $* \mathrm{QS}$.

(2) For the MHES networks, an index 1 is used to represent the networks that were generated using disaggregated data. For networks generated with aggregated data, we used an index 2. For example, 2015.1 and 2015.2 are the networks that represent the relationship between the universities in 2015, according to the disaggregated and aggregated data, respectively.

(3) The multiplex networks are formed by a set of monoplex networks. For example, for MHES multiplex networks, 2013-2015.2 is the multiplex network formed by the 2013.2, 2014.2, and 2015.2 MHES monoplex networks, and for QSRanking networks, 2018-2020.QS is the multiplex network formed by 2018.QS, 2019.QS, and 2020.QS QSRanking monoplex networks.

In Table 3, we present the number of nodes and links for each layer.

As mentioned above, the QSRanking and MHES networks have a large number of links in each layer regarding the number of nodes. Therefore, these networks can help us verify how efficient our methodology is.

Now, in order to see the relationships between universities as shown in Figure 1, the 2-layer multiplex network is modeled with the disaggregated data for the years 2013 and 2014, and Figure 2 shows the 3-layer network modeled with the aggregated data for the years 2015, 2016, and 2017.

Here, it is important to mention that, in each multiplex network, the nodes represent universities in the MHES, and the intralayer links represent their relationships for a specific year. The interlayer links represent the connection of replica nodes in different years (same nodes in other layers).

For example, in Figure 2, the links in layers 11, 12, and 13 represent the relationships of each pair of universities in the years 2015, 2016, and 2017, respectively, while the links that join 11 with 12 and 12 with 13 are the identity relationship.

Since the same universities in different layers have different relationships, finding which nodes are essential to remain the multiplex networks connected is not a trivial problem.

\subsection{Inverse Percolation through the Adaptation of Vertex} Separator Problem ( $m$-VSP). As mentioned above, the robustness in a network can be quantified, finding the nodes that cause the rupture of the GC (inverse percolation process). In multiplex networks, we can identify the GC as the MCGC or the LVC, which are defined as [43]
(1) Mutually connected giant component: each node $i$ is in the MCGC if it has at least one neighbor $j$ that belongs to the MCGC and if all its replica nodes in each interdependent network are also in the MCGC.

From this definition, Bianconi et al. [43] deduce that if a node $i$ in a certain layer of a connected network of networks is in the MCGC, then all its replica nodes are also in the MCGC.

(2) Large viable component: viability can be seen as the existence of several paths, connecting any pair of nodes in each of the layers of the entire network. By definition, an LVC is a set of nodes interconnected with each other at the same time on all individual layers $[25,44]$ of maximal size.

This makes an LVC at most the size of the intersection of the set of connected nodes at each network layer.

On the other hand, the methodology presented in this work is defined as an adaptation of the methodology denoted in [21], which is based on the resolution of the vertex separator problem (VSP) [45]. Specifically, an m-VSP consists in finding a minimum set of nodes $C$ that, being deleted from the network, it produces a disconnection of the MCGC and the LVC of the multiplex network into two connected components $(A, B)$, in such a way that $A$ and $B$ are maximized. Formally, the m-VSP can be summarized as:

(i) Instance: a MCGC or a LVC of a multiplex network $\mathrm{GP}=\left(G_{\alpha}, C\right)$.

(ii) Problem: to find a partition of the MCGC or the LVC that results into three disjoint sets $A, B$ and $C$, and $A$ and $B$ nonempty, such that

P1) There is no interlayer or intralayer links between the elements belonging to each set $A, B$, or $C$ P2) $A$ and $B$ are maximized

P3) $C$ is minimized

Thus, if we solve the m-VSP on a multiplex network, we obtain nodes that cause the rupture of the MCGC or LVC. Therefore, we can assure that the resolution of the m-VSP is an inverse percolation process and finds the set of relevant nodes for network communication.

It is important to mention that we can quantify the robustness of the networks by analyzing the percentage of nodes that belong to the separator set $C$ of the MCGC and LVC. For example, for MCGC, a high percentage (more than $70 \%$ ) of nodes in $C$ indicates that the network is robust, while a low percentage (less than 30\%) indicates that the network is not very robust, and for LVC, a high percentage (more than $60 \%$ ) of nodes in $C$ indicates that the network is robust, while a low percentage (less than $40 \%$ ) indicates that the network is not very robust.

3.4. Simulated Annealing to Solve $m$-VSP. Because the original VSP is considered NP-hard [46, 47], the adaptation from multiplex networks is NP-hard too [48, 49]; therefore, we can solve the adaptation of VSP using a heuristic method. 
TABLE 3: Main characteristics for MHES and QSRanking networks.

\begin{tabular}{lcccc}
\hline Network & \multicolumn{5}{c}{ Nodes } & 13 & 11,12 , and 13 \\
\hline $2013-2014.1$ & 1214 & 1144 & - & 60 \\
$2014-2015.1$ & 1144 & 1210 & - & 60 \\
$2015-2016.1$ & 1210 & 1036 & - & 60 \\
$2016-2017.1$ & 1036 & 1018 & - & 60 \\
$2013-2015.1$ & 1214 & 1022 & 1210 & 60 \\
$2014-2016.1$ & 1144 & 1210 & 1036 & 60 \\
$2015-2017.1$ & 1210 & 1036 & 1018 & 60 \\
$2013-2014.2$ & 1130 & 1154 & - & 60 \\
$2014-2015.2$ & 1154 & 1172 & - & 60 \\
$2015-2016.2$ & 1172 & 920 & - & 60 \\
$2016-2017.2$ & 920 & 1008 & - & 60 \\
$2013-2015.2$ & 1130 & 1154 & 1172 & 60 \\
$2014-2016.2$ & 1154 & 1172 & 920 & 60 \\
$2015-2017.2$ & 1172 & 920 & 1008 & 60 \\
$2018-2019 . Q S$ & 9648 & 11426 & - & 377 \\
$2019-2020 . Q S$ & 11426 & 10668 & - & 377 \\
$2018-2019 . Q S$ & 9648 & 11426 & 10668 & 377 \\
\hline
\end{tabular}

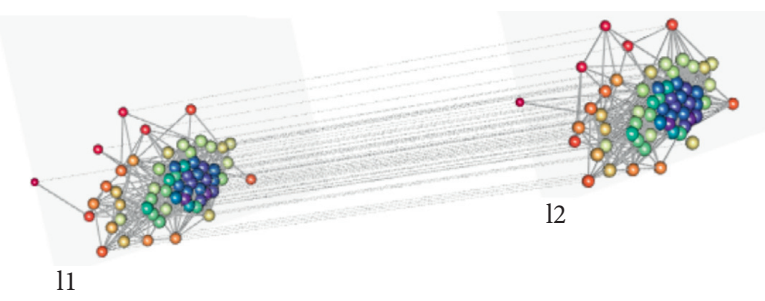

FIGURE 1: Graph of the MHES network with disaggregated data for the years 2013 and 2014 .

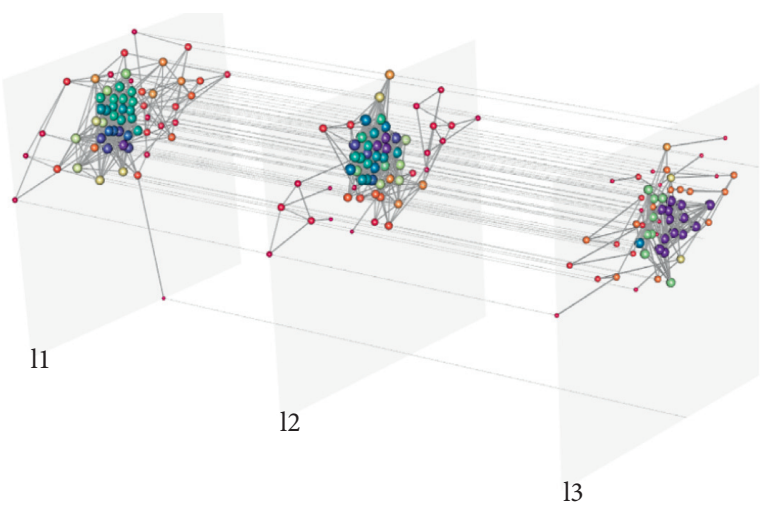

FIGURE 2: Graph of the MHES network with nonstandardized disaggregated data for the years 2015, 2016, and 2017.

In this work, we used a simulated annealing algorithm (SA) [50] developed in C language. SA is a technique based on local search, and it is necessary to establish the structure of neighborhoods. In the development of this work, two neighborhoods were used: Hamming distance 1 (Hamming distance is defined as the number of elements that have to be changed to transform a solution into another valid solution) and Hamming distance 2. For the first type of neighborhood, it is added or deleted one node of the current solution with the following steps:

(1) A random number between 0 and 1 is generated

(2) If random $\leq 0.5$, one node is removed; otherwise, one node is added

(3) To remove an element, it is chosen randomly, giving the same probability to each element that belongs to the current solution

(4) To add an element, it is chosen randomly, giving the same probability to each element that does not belong to the current solution

For the second neighborhood, two nodes are removed, or two nodes are added, and if it is the case that two nodes cannot be removed or added, one is deleted, and a different one is added. With this, the distance of Hamming 2 is maintained.

Finally, the algorithm finished when the best solution it is not updated, when 50 temperature updates have elapsed, or $T_{k}=T_{f}$.

SA requires 4 control parameters, and in order to obtain a good performance, it is important to find the effective parameter settings for the technique. Then, using the differential evolution algorithm (DE) [51]; we obtain the following values (for more information, see [10]):

(i) Initial temperature $T_{i}=1500$

(ii) Final temperature $T_{f}=0.001$

(iii) Cooling program $T_{k} \gamma$, with $\gamma=0.95$

(iv) Times that a new neighbor is generated $L_{k}=20$

\section{Results and Discussion}

In this section, we show the numerical results for the quantification of robustness in multiplex networks based on the $\mathrm{m}$-VSP.

4.1. Benchmark Networks. To verify if the proposed methodology can be applied in different types and models of networks, in this section, we present an analysis of quantification of robustness in different 3-layer multiplex networks.

At this point, it is important to mention that the robustness analysis in multiplex networks is an emerging research area, and we could not find an appropriate benchmark set in the specific literature.

Therefore, we decided to use a set of benchmark networks (Tables 1 and 2) that have been used for different purposes, such as the identification of communities and the analysis of their structural properties [52-54].

For example, there are some methodologies proposed in the specific literature, such as $[16,22-24]$, in which, the authors do not carry out analysis or experiments in benchmark networks since they focus only on their own case studies.

In Table 4, we present the numerical values obtained by applying the m-VSP to the MCGC, while in Table 5, we show 
TABLE 4: Numerical results for quantification of robustness in benchmark networks (MCGC).

\begin{tabular}{lccc}
\hline Network & $C$ & $A$ and $B$ & \% of nodes of MCGC in $C$ \\
\hline CKM & 50 & 23 and 28 & 47.61 \\
Hepatitis & 1 & - & 1.25 \\
Krackhardt & 6 & 6 and 1 & 28.57 \\
Lazega & 39 & 21 and 2 & 54.92 \\
\hline
\end{tabular}

TABLE 5: Numerical results for quantification of robustness in benchmark networks (LVC).

\begin{tabular}{lccc}
\hline Network & $C$ & $A$ and $B$ & \% of nodes of LVC in $C$ \\
\hline CKM & 19 & 19 and 17 & 18.26 \\
Hepatitis & 1 & - & 1.25 \\
Krackhardt & 6 & 6 and 1 & 28.57 \\
Lazega & 21 & 19 and $12 \%$ & 29.57 \\
\hline
\end{tabular}

the numerical values obtained by applying the $\mathrm{m}$-VSP to the LVC. In these tables, in the first column, we show the identifier for each network; in the second column, the values for $C$; in the third column, the values for $A$ and $B$; and in the fourth column, the percentage of nodes in MCGC that belong to $C$.

It is important to remember that if $C$ has a value greater than $70 \%$ of the total nodes of the MCGC or the $60 \%$ of the LVC, it is considered robust. On the other hand, when $C$ has a value lower than $30 \%$ for MCGC or $40 \%$ for LVC, we can affirm that the network is not very robust.

Based on the information of Table 4, we can see that, from Krackhardt and Lazega, the percentage of nodes of the LVC in set $C$ are moderately robust since each network has a $C$ of $28.57 \%$ and $54.92 \%$, respectively.

In the specific case of the Hepatitis network, there is only one node in the separator set; and the subsets $A$ and $B$ cannot be formed since each node that belongs to the network remains as an isolated component, and it is very prone to collapse.

Based on the information of Table 5, we can see that, from Krackhardt and Lazega, the percentage of nodes of the LVC in set $C$ are not robust since each network has a $C$ of $28 \%$ and $29.57 \%$, respectively.

As in the case of the analysis using the MCGC, for the Hepatitis network, there is only one node in the separator set, and the subsets $A$ and $B$ cannot be formed since each node that belongs to the network remains as an isolated component. These results are produced because the network has a star structure, and it is very prone to collapse. On the other hand, the CKM network has a separator set $C$ of 19 nodes that are around $18 \%$ of the total nodes. Therefore, both networks are not very robust.

Therefore, based on the above, we can see that the $\mathrm{m}$-VSP can find the nodes important to maintaining the communication and the correct operation of the systems.

4.2. QSRanking and MHES Networks. Based on the previous section results, we can see that the proposed methodology works for different types of network models (applied to its MCGC and LVC). Therefore, we present the structural metrics analysis and quantification of robustness for the QSRanking and MHES networks.

4.2.1. Structural Metrics. In Table 6, we present the numerical results for the structural metrics in the monoplex HESM networks modeled using the disaggregated and aggregated data and in the QSRanking monoplex networks, where we can see that, from QSRanking networks, the average degree is around a quarter part of the total number of nodes. For the MHES, the average degree value for networks modeled with aggregated data is smaller than for networks modeled using disaggregated data; however, the maximum degree in most networks (except 2016.2) is around half of the total network nodes.

We can see that all networks have a high clustering coefficient (between 0.6 and 0.9) and have a small path length between any pair of nodes (between 2 and 3). Therefore, we can deduce that RankingQS and MHES networks follow the small-world model [28], which implies that they comply with the main characteristics of social networks (SN) [36].

4.2.2. Quantification of Robustness. In this section, we present the numerical results obtained by solving the $\mathrm{m}$-VSP to the MCGC and the LVC for each QSRanking and MHES network; Table 7 presents the cardinality value for sets $A, B$, and $C$ and the percentage of nodes of MCGC that belong to C. Here, it is important to mention that, in the QSRanking and MHES networks, the MCGC is formed by all the nodes of the networks.

According to the results shown in Table 7, we can assure that QSRanking and MHES networks are moderately robust; since for all QSRanking networks, $C$ is between $34 \%$ and $46 \%$, and for all MHES networks, $C$ is between $20 \%$ and $46 \%$ of the total nodes belonging to the MCGC. For example, for the networks 2013.1-2014.1 and 2018-2020.QS, we have 23\% and $46 \%$ of the nodes in the separator set, respectively.

Now, we present the numerical results obtained by solving the m-VSP to the LVC for each QSRanking and MHES network; Table 8 presents the cardinality value for sets $A, B$, and $C$ and the percentage of nodes of LVC that belong to $C$. Here, it is important to mention that the size of the LVC for each network is provided in Table 8.

Based on Table 8, we can see that, for the MHES and QSRanking networks, the LVC is smaller compared to the MCGC. This is because the MCGC is composed of the union, and the LVC is composed at most by the union of all the giant components of every single layer.

According to the results shown in Table 9, we can assure that QSRanking and MHES networks are not robust; since for all QSRanking networks, $C$ is between 28 and $33.8 \%$ and for all MHES networks, $C$ is between $16.9 \%$ and $36.7 \%$ of the total nodes belonging to the LVC. For example, for the networks 2016.1-2017.1 and 2019-2020.QS, we have 31.3\% and $35.2 \%$ of the nodes in the separator set, respectively. 
TABLE 6: Numerical values for structural metrics in QSRanking MHES monoplex networks.

\begin{tabular}{lcccc}
\hline Network & Average degree & Max. degree & Clustering & Path length \\
\hline 2013.1 & 18.276 & 30 & 0.632 & 1.882 \\
2014.1 & 18.508 & 30 & 0.611 & 1.885 \\
2015.1 & 18.4 & 30 & 0.6888 & 1.874 \\
2016.1 & 16.103 & 30 & 0.574 & 1.966 \\
2017.1 & 16.237 & 30 & 0.574 & 2.113 \\
2013.2 & 15.733 & 30 & 0.618 & 2.106 \\
2014.2 & 14.533 & 30 & 0.629 & 2.325 \\
2015.2 & 14.678 & 30 & 0.634 & 2.635 \\
2016.2 & 5.786 & 13 & 0.716 & 3.757 \\
2017.2 & 15.017 & 27 & 0.722 & 2.56 \\
2018. QS & 91.358 & 109 & 0.858 & 1.13 \\
2019. QS & 198.713 & 110 & 0.863 & 1.28 \\
$2020 . Q S$ & 96.108 & 109 & 0.865 & 1.26 \\
\hline
\end{tabular}

TABLe 7: Numerical results for m-VSP for QSRanking and MHES networks (MCGC).

\begin{tabular}{lccc}
\hline Network & $C$ & $A$ and $B$ & \% of nodes of MCGC in $C$ \\
\hline $2013-2014.1$ & 14 & 28 and 18 & 23.3 \\
$2014-2015.1$ & 13 & 24 and 23 & 21.7 \\
$2015-2016.1$ & 15 & 27 and 18 & 25 \\
$2016-2017.1$ & 19 & 25 and 16 & 31.7 \\
$2013-2015.1$ & 24 & 18 and 18 & 40 \\
$2014-2016.1$ & 27 & 21 and 12 & 45 \\
$2015-2017.1$ & 24 & 19 and 17 & 40 \\
$2013-2014.2$ & 18 & 27 and 15 & 30 \\
$2014-2015.2$ & 14 & 27 and 19 & 33.3 \\
$2015-2016.2$ & 15 & 25 and 20 & 25 \\
$2016-2017.2$ & 19 & 29 and 16 & 31.7 \\
$2013-2015.2$ & 26 & 19 and 15 & 43.3 \\
$2014-2016.2$ & 24 & 20 and 16 & 40 \\
$2015-2017.2$ & 26 & 18 and 16 & 43.3 \\
$2018-2019 . Q S$ & 112 & 137 and 128 & 34 \\
$2019-2020 . Q S$ & 133 & 137 and 107 & 35.3 \\
$2018-2020 . Q S$ & 174 & 108 and 95 & 46.1 \\
\hline
\end{tabular}

Furthermore, we can verify that the methodology proposed in this work can find the set of nodes that cause the rupture of the MCGC and the LVC for different models of 2layer and 3-layer multiplex networks since the sum of $A, B$, and $C$ is equivalent to the total number of nodes that belong to the MCGC or the LVC according to the case.

It is important to mention that, for MHES networks, there is no difference in the networks modeled with both disaggregated and aggregated data, so they have the same numerical results when $\mathrm{m}$-VSP is applied.

The above means that both types of networks are equivalent and imply that it is possible to use a reduced set of information to analyze the behavior of the MHES; therefore, we can assure that the main characteristics of the complex system are properly structured when we modeled the QSRanking and MHES networks.

Then, we can verify that the methodology proposed in this work can find the set of nodes that cause the rupture of
TABLE 8: Size of LVC for each MHES and QSRanking network.

\begin{tabular}{lccc}
\hline Network & Size of LVC & Network & Size of LVC \\
\hline $2013-2014.1$ & 53 & $2015-2016.2$ & 49 \\
$2014-2015.1$ & 47 & $2016-2017.2$ & 50 \\
$2015-2016.1$ & 49 & $2013-2015.2$ & 51 \\
$2016-2017.1$ & 51 & $2014-2016.2$ & 46 \\
$2013-2015.1$ & 53 & $2015-2017.2$ & 52 \\
$2014-2016.1$ & 47 & $2018 . Q S$ & 319 \\
$2015-2017.1$ & 49 & $2019 . Q S$ & 324 \\
$2013-2014.2$ & 51 & $2020 . Q S$ & 328 \\
$2014-2015.2$ & 47 & - & - \\
\hline
\end{tabular}

TABLE 9: Numerical results for m-VSP for QSRanking and MHES networks (LVC).

\begin{tabular}{lccc}
\hline Network & $C$ & $A$ and $B$ & \% of nodes of LVC in $C$ \\
\hline $2013.1-2014.1$ & 9 & 26 and 25 & 16.9 \\
$2014.1-2015.1$ & 11 & 26 and 23 & 23.4 \\
$2015.1-2016.1$ & 12 & 28 and 20 & 24 \\
$2016.1-2017.1$ & 16 & 23 and 21 & 31.3 \\
$2013.1-2015.1$ & 18 & 22 and 20 & 33.9 \\
$2014.1-2016.1$ & 12 & 25 and 23 & 25.5 \\
$2015.1-2017.1$ & 18 & 22 and 20 & 36.7 \\
$2013.2-2014.2$ & 17 & 23 and 20 & 33.3 \\
$2014.2-2015.2$ & 13 & 27 and 19 & 27.6 \\
$2015.2-2016.2$ & 12 & 25 and 23 & 24.4 \\
$2016.2-2017.2$ & 13 & 29 and 18 & 26 \\
$2013.2-2015.2$ & 18 & 22 and 20 & 35.3 \\
2014.2-2016.2 & 16 & 22 and 22 & 34.8 \\
2015.2-2017.2 & 16 & 23 and 21 & 30.7 \\
2018.QS-2019.QS & 108 & 142 and 127 & 33.8 \\
2019.QS-2020.QS & 114 & 137 and 126 & 35.2 \\
2018.QS-2020.QS & 92 & 149 and 136 & 28
\end{tabular}

the MCGC or the LVC for different models of 2-layer and 3layer multiplex networks.

In order to analyze the elements that cause the rupture of the MCGC and the LVC in the QSRanking and MHES networks, based on the results presented in $[8,28]$ about the classification of universities, we perform an analysis.

In Table 10, we show the percentage of the 377 universities that we consider to model the QSRanking networks that are located between positions 1 and 100; 101 and 200; 201 and 300; 301 and 400; and 401 and 500 in the main university rankings (like the QS ranking).

Based on the information presented in Table 10, we can see that most of the universities that cause the rupture of the network are between positions 100 and 300 of the QS ranking, and their main characteristics are as follows: average reputation, high employer reputation, average number of citations, few international faculties, and a greater number of national students.

Now, in Table 11, we show the percentage of universities with high, average, and low values in the university rankings that cause the disconnection of the MHES.

Based on the results of Table 11, we can see that, for all MHES networks, the percentage of universities with high-, average-, and low-ranking values is divided equally, that is, a 
TABLE 10: Percentage of universities in 100-universities ranges (QSRanking).

\begin{tabular}{lccccc}
\hline Network & $1-100(\%)$ & $101-200(\%)$ & $201-300(\%)$ & $301-400$ & $401-500(\%)$ \\
\hline $2018-2019 . Q S$ & 20.7 & 29.3 & 28.4 & 28.7 & 15.2 \\
$2019-2020 . Q S$ & 18.4 & 29.3 & 30 & 6.4 & $14 \%$ \\
$2018-2020 . Q S$ & 19.5 & 27.5 & 9.1 & 9 \\
\hline
\end{tabular}

TABle 11: Percentage of universities with high, average, and low-ranking value (MHES).

\begin{tabular}{lccc}
\hline Network & High ranking (\%) & Average ranking (\%) & Low ranking $(\%)$ \\
\hline $2013.1-2014.1$ & 35.7 & 28.6 & 35.7 \\
$2014.1-2015.1$ & 30.4 & 23.3 & 46.3 \\
$2015.1-2016.1$ & 40 & 33.3 & 26.6 \\
$2016.1-2017.1$ & 36.6 & 26.6 & 36.6 \\
$2013.1-2015.1$ & 33.3 & 29 & 37.5 \\
$2014.1-2016.1$ & 29.6 & 40.7 & 29.6 \\
$2015.1-2017.1$ & 33 & 25 & 41.6 \\
$2013.1-2014.2$ & 33.3 & 22.2 & 44.4 \\
$2014.1-2015.2$ & 35.7 & 28.6 & 35.7 \\
$2015.1-2016.2$ & 40 & 33.3 & 26.7 \\
$2016.1-2017.2$ & 42.1 & 26.3 & 31.6 \\
$2013.1-2015.2$ & 34.6 & 30.7 & 34.6 \\
$2014.1-2016.2$ & 33.3 & 29.1 & 34.6 \\
$2015.1-2017.2$ & 38.4 & 30.8 & 30.8 \\
\hline
\end{tabular}

similar amount of the three types of universities are necessary to cause the rupture of the LVC.

From a social network perspective, we can deduce that the links between the different types of universities are essential for the correct performance and development of the QSRanking and MHES networks.

Finally, it is important to mention that the proposed methodology obtains a set of nodes that cause the rupture of the MCGC and the LVC without using structural network information or centrality measures.

\section{Limitations of the Study and Discussion}

The analysis developed in this work is based on the analysis of the rupture of the MCGC or the LVC belonging to each multiplex network. The idea of using the two ways is because the MCGC can give us an idea of the aggregated network; on the other hand, the LVC can give us the information about the nodes that are very important in the behavior of each layer.

The idea of modeling two or three different types of layers for the MHES and QSRanking networks is to be able to obtain an identification of the best universities, based on a multicriteria analysis that reduces the disadvantages of using only one set of nodes; thus, the technique is capable of identifying those universities that, based on different characteristics, are the most important for the operation of the Mexican and international higher education system.

We apply the multiplex network approach using the adaptation of the vertex separator problem to identify those nodes based on their main characteristics, when their links are eliminated from all the layers of the network, causing the rupture of the system.

Therefore, a limitation of the study is that the drastic change of one or more variables by year changes the dynamics of the system.

\section{Conclusions}

This paper presents the adaptation of the vertex separator problem (VSP) for multiplex networks, denoted as m-VSP, which using inverse percolation obtains the nodes that, when removed from the system, cause the rupture of the mutually connected giant component (MCGC) or the large viable cluster (LVC).

Also, based on the tests performed in this work, we can deduce that the main advantage of using $\mathrm{m}$-VSP is that no structural calculations or centrality metrics are needed.

Numerical results show that the m-VSP can work on different types of multiplex networks and that m-VSP can get a set of nodes that cause the rupture of the MCGC or the LVC.

For QSRanking networks, the results show that most universities that cause the rupture of the LVC are between positions 101 and 300 in the ranking, and few universities are between positions 0-100 or 401-500.

On the other hand, in the specific case of MHES networks, the results show that most universities that cause the rupture of the LVC are divided into three types: universities that obtain high, average, or low values in the university rankings.

As future work, we propose the implementation of intelligence-based techniques of self-adaptive swarms and/or exact methods for solving the mathematical model m-VSP.

\section{Data Availability}

The data used to support the findings of this work are available from the corresponding author upon request.

\section{Conflicts of Interest}

The authors declare no conflicts of interest. 


\section{References}

[1] G. D'Agostino and A. Scala, Networks of Networks: The Last Frontier of Complexity, Springer, Berlin, Germany, 2014.

[2] H. N. Temperley and E. H. Lieb, "Relations between the percolation and coloring problem and other graph-theoretical problems associated with regular planar lattices: some exact results for the percolation problem," Proceedings of the Royal Society of London. A. Mathematical and Physical Sciences, vol. 322, no. 1549, pp. 251-280, 1971.

[3] G. Cencetti, F. Bagnoli, G. Battistelli, L. Chisci, and D. Fanelli, "Control of multidimensional systems on complex network," PLoS One, vol. 12, no. 9, Article ID e0184431, 2017.

[4] E. Cozzo, A. Arenas, Y. Moreno et al., "Clustering coefficients in multiplex networks," 2013, http://arxiv.org/abs/1307.6780, Article ID 073029.

[5] K.-M. Lee, B. Min, and K.-I. Goh, "Towards real-world complexity: an introduction to multiplex networks," The European Physical Journal B, vol. 88, no. 2, p. 48, 2015.

[6] R. J. Mondragon, J. Iacovacci, and G. Bianconi, "Multilink communities of multiplex networks," PloS One, vol. 13, no. 3, Article ID e0193821, 2018.

[7] P. J. Mucha, T. Richardson, K. Macon, M. A. Porter, and J.-P. Onnela, "Community structure in time-dependent, multiscale, and multiplex networks," Science, vol. 328, no. 5980, pp. 876-878, 2010.

[8] Unit QI, "Qs intelligence unit-international indicators," in International Indicators, pp. 327-395, Unit, Q. I, England, UK, 2017.

[9] U. ExECUM-Estudio Comparativo de Universidades, Dirección general de evaluación institucional, UNAM, Mexico City, Mexico, 2020, http://www.execum.unam.mx.

[10] E. Montes-Orozco, R. A. Mora-Gutiérrez, B. ObregónQuintana et al., "Mexican university ranking based on maximal clique," in Educational Networking, pp. 327-395, Springer, Berlin, Germany, 2020.

[11] B. Min and M. Zheng, "Correlated network of networks enhances robustness against catastrophic failures," PloS One, vol. 13, no. 4, Article ID e0195539, 2018.

[12] A. Nagurney and Q. Qiang, "Fragile networks: identifying vulnerabilities and synergies in an uncertain age," International Transactions in Operational Research, vol. 19, no. 1-2, pp. 123-160, 2012.

[13] M. E. J. Newman, "The structure and function of complex networks," SIAM Review, vol. 45, no. 2, pp. 167-256, 2003.

[14] M. Ouyang, L. Hong, Z.-J. Mao, M.-H. Yu, and F. Qi, “A methodological approach to analyze vulnerability of interdependent infrastructures," Simulation Modelling Practice and Theory, vol. 17, no. 5, pp. 817-828, 2009.

[15] V. Latora and M. Marchiori, "Vulnerability and protection of infrastructure networks," Physical Review E, vol. 71, no. 1, Article ID 015103, 2005.

[16] C. M. Schneider, A. A. Moreira, J. S. Andrade, S. Havlin, and H. J. Herrmann, "Mitigation of malicious attacks on networks," Proceedings of the National Academy of Sciences, vol. 108, no. 10, pp. 3838-3841, 2011.

[17] H. Zhang, E. Fata, and S. Sundaram, "A notion of robustness in complex networks," IEEE Transactions on Control of Network Systems, vol. 2, no. 3, pp. 310-320, 2015.

[18] X.-L. Ren, N. Gleinig, D. Toli_c, and N. Antulov-Fantulin, "Underestimated cost of targeted attacks on complex networks," Complexity, vol. 2018, 2018.

[19] J. Zhang, S. Wang, and Y. Wang, "Optimal design on robustness of scale-free networks based on degree distribution," Scientific Programming, vol. 2016, 2016.
[20] F. Morone and H. A. Makse, "Influence maximization in complex networks through optimal percolation," Nature, vol. 524, no. 7563, pp. 65-68, 2015.

[21] E. Montes-Orozco, R.-A. Mora-Gutiérrez, S.-G. De-LosCobos-Silva et al., "Methodology to quantify the robustness in networks. Case study: higher education system in Mexico," in Computing, Page ForthcomingSpringer, Berlin, Germany, 2020.

[22] L. M. Shekhtman, M. M. Danziger, and S. Havlin, "Recent advances on failure and recovery in networks of networks," Chaos, Solitons \& Fractals, vol. 90, pp. 28-36, 2016.

[23] M. De Domenico, V. Nicosia, A. Arenas, and V. Latora, "Structural reducibility of multilayer networks," Nature Communications, vol. 6, no. 1, pp. 1-9, 2015.

[24] K.-K. Kleineberg, L. Buzna, F. Papadopoulos, M. Boguñá, and M._A. Serrano, "Geometric correlations mitigate the extreme vulnerability of multiplex networks against targeted attacks," Physical Review Letters, vol. 118, no. 21, p. 218301, 2017.

[25] G. J. Baxter, G. Bianconi, R. A. da Costa, S. N. Dorogovtsev, and J. F. F. Mendes, "Correlated edge overlaps in multiplex networks," Physical Review E, vol. 94, Article ID 012303, 2016.

[26] G. Bianconi, "Dangerous liaisons?" Nature Physics, vol. 10, no. 10, pp. 712-714, 2014.

[27] A. Aleta, S. Meloni, and Y. Moreno, "A multilayer perspective for the analysis of urban transportation systems," Scientic Reports, vol. 7, Article ID 44359, 2017.

[28] D. Vaknin, M. M. Danziger, and S. Havlin, "Spreading of localized attacks in spatial multiplex networks," New Journal of Physics, vol. 19, no. 7, Article ID 073037, 2017.

[29] M. Stella, "Multiplex networks quantify robustness of the mental lexicon to catastrophic concept failures, aphasic degradation and ageing," Physica A: Statistical Mechanics and Its Applications, vol. 554, p. 124382, 2020.

[30] S. Osat, A. Faqeeh, and F. Radicchi, "Optimal percolation on multiplex networks," Nature Communications, vol. 8, no. $1547,2017$.

[31] E. Montes-Orozco, R.-A. Mora-Gutiérrez, S.-G. De-LosCobos-Silva et al., "Identification of COVID-19 spreaders using multiplex networks approach," IEEE Access, vol. 8, pp. 122874-122883, 2020.

[32] M. De Domenico, M. A. Porter, and A. Arenas, "Muxviz: a tool for multilayer analysis and visualization of networks," Journal of Complex Networks, vol. 3, no. 2, pp. 159-176, 2015.

[33] J. Coleman, E. Katz, and H. Menzel, "The diffusion of an innovation among Physicians," Sociometry, vol. 20, no. 4, pp. 253-270, 1957.

[34] C. Stark, B.-J. Breitkreutz, T. Reguly, L. Boucher, A. Breitkreutz, and M. Tyers, "Biogrid: a general repository for interaction datasets," Nucleic Acids Research, vol. 34, no. suppl 1, pp. D535-D539, 2006.

[35] D. Krackhardt, "Cognitive social structures," Social Networks, vol. 9, no. 2, pp. 109-134, 1987.

[36] E. Lazega et al., The Collegial Phenomenon: The Social Mechanisms of Cooperation Among Peers in a Corporate Law Partnership, Oxford University Press on Demand, Oxford, UK, 2001.

[37] D. J. Watts and S. H. Strogatz, "Collective dynamics of 'smallworld' networks," Nature, vol. 393, no. 6684, p. 440, 1998.

[38] P. Erdös and A. Rényi, "On the evolution of random graphs," Publications of the Mathematical Institute of the Hungarian Academy of Sciences, vol. 5, no. 1, pp. 17-60, 1960.

[39] R. Albert and A.-L. Barabási, "Statistical mechanics of complex networks," Reviews of Modern Physics, vol. 74, no. 1, p. $47,2002$. 
[40] R. Albert, H. Jeong, and A.-L. Barabási, "Error and attack tolerance of complex networks," Nature, vol. 406, no. 6794, pp. 378-382, 2000.

[41] C. Gockel and L. Werth, "Measuring and modeling shared leadership: Traditional approaches and new ideas," Journal of Personnel Psychology, vol. 9, no. 4, pp. 172-180, 2010.

[42] P. C. Mahalanobis, On the Generalized Distance in Statistics, National Institute of Science of India, Odisha, India, 1936.

[43] G. Bianconi and F. Radicchi, "Percolation in real multiplex networks," Physical Review E, vol. 94, no. 6, Article ID 060301, 2016.

[44] M. Stella and Y. N. Kenett, "Viability in multiplex lexical networks and machine learning characterizes human creativity," Big Data and Cognitive Computing, vol. 3, p. 124382, 2019.

[45] E. Balas and C. C. d. Souza, "The vertex separator problem: a polyhedral investigation," Mathematical Programming, vol. 103, no. 3, pp. 583-608, 2005.

[46] R. Müller and D. Wagner, " $\alpha$-Vertex separator is NP-hard even for 3-regular graphs," Computing, vol. 46, no. 4, pp. 343-353, 1991.

[47] F. Rendl and R. Sotirov, "The min-cut and vertex separator problem," Computational Optimization and Applications, vol. 69, no. 1, pp. 159-187, 2018.

[48] J. Dréo, A. P_etrowski, P. Siarry, and E. Taillard, Metaheuristics for Hard Optimization: Methods and Case Studies, Springer Science \& Business Media, Berlin, Germany, 2006.

[49] M. R. Garey, D. S. Johnson, and R. Sethi, "The complexity of flowshop and jobshop scheduling," Mathematics of Operations Research, vol. 1, no. 2, pp. 117-129, 1976.

[50] S. Kirkpatrick, C. D. Gelatt, and M. P. Vecchi, "Optimization by simulated annealing," Science, vol. 220, no. 4598, pp. 671-680, 1983.

[51] R. Storn and K. Price, "Differential evolution-a simple and efficient heuristic for global optimization over continuous spaces," Journal of Global Optimization, vol. 11, no. 4, pp. 341-359, 1997.

[52] E. Cozzo, G. F. de Arruda, F. A. Rodrigues, and Y. Moreno, "Multilayer networks: metrics and spectral properties," in Interconnected Networks, pp. 17-35, Springer, Berlin, Germany, 2016.

[53] M. T. Heaney, "Multiplex networks and interest group influence reputation: an exponential random graph model," Social Networks, vol. 36, pp. 66-81, 2014.

[54] A. Reiffers-Masson and V. Labatut, "Opinion-based centrality in multiplex networks: a convex optimization approach," 2017, http://arxiv.org/abs/1703.03741. 